American Journal of Applied Sciences 8 (12): 1287-1294, 2011

ISSN 1546-9239

(C) 2011 Science Publications

\title{
A Method for Human Resource Risk Management in Mobile Workforce Brokering Systems
}

\author{
Arash Mousavi, Md. Jan Nordin, Zulaiha Ali Othman and Riza Sulaiman \\ School of Computer Science, Faculty of Information Science and Technology, \\ Universiti Kebangsaan Malaysia, 43600, Bangi, Malaysia
}

\begin{abstract}
Problem statement: Human Resource Risk Management is one of the crucial issues in Mobile Workforce Management Systems (MWM) in general, and in Mobile Workforce Brokering Systems (MWBS) in Particular. It is important because, if not properly managed, it will cause reduction in accuracy of the automated MWBS, which in turn necessitates more human involvement in the task allocation process. Thus, no reliable planning and scheduling schema can be made or achieved. Approach: However, a proven approach to tackle this problem is via contingency planning. In this study, we examined a specific type of HR risk called Unexpected Absence of mobile workforces in the context of an ontology-driven and multiagent-based MWBS. Our contingency plan that mainly consists of a statistical method is incorporated into the body of a coordination medium represented in OWL ontology format. Results: The proposed statistical method evaluates the past history and the current claims of an MW in order to find out a realistic plan for the next period of the system's run. Conclusion: Finally, via a case study we have illustrated that this method increases the accuracy and reliability of a periodical plan, made for MWBS in its initialization phase.
\end{abstract}

Key words: Mobile Workforce Brokering Systems (MWBS), Human Resource (HR), Mobile Workforces (MW), Mobile Workforce Brokering System (MWBS), Task Allocation Agent (TAA), processes performed

\section{INTRODUCTION}

Addressing Human Resource (HR) risks such as: emergency leave, unexpected absence or resignation is a determinative activity in successful execution of business processes performed by workforces of an organization. However, according to (Malone and Crowston, 1990; Kappel et al., 1998; Pal et al., 2001) HR risks are less regarded in comparison with other types of risks such as financial or safety. Managing HR risks is important because firstly, workforces are the key players in every organization and secondly, any disruption in task performance caused by HR risks will have negative impact on overall performance of the companies in which they are employed. Similarly, in the context of an automated Mobile Workforce Brokering System (MWBS) (Mousavi et al., 2010a; 2010b; Mousavi and Nordin, 2007; Mousavi et al., 2011), where task allocation has to follow a prescheduled pattern, any unexpected or unplanned unavailability of Mobile Workforces (MW) will cause reduction in overall performance of their corresponding organization.
Previous works: According to (Wang et al., 2005) business organizations in the past, paid more attention to commercially available and proven technological solution in comparison with the commercial conditions, in adapting a new technology to their business processes. However, this technology-driven approach lacks adequate knowledge about the actual benefits that a new technology can bring to organizations (Meijer et al., 2002). Therefore, utilizing mobile technology and particularly mobile workforces cannot be an effective plan without investigating its pros and cons. Although mobile workforce solution has many positive impacts on different aspects of an organization (Wang et al., 2006), it can also bring harm and loss to the organization without precise investigation on failure factors in adapting it. In this direction, risk is one of the main issues in adapting workforces to mobile technology (Meijer et al., 2002).

Risk in MWM (Chiu et al., 2005) has been taken into consideration by some researchers. For instance as described in (Shi-Cho et al., 2005a; 2005b), MWs specially field workers may face different kind of circumstances in the field. These circumstances, which

Corresponding Author: Arash Mousavi, School of Computer Science, Faculty of Information Science and Technology,

University Kebangsaan Malaysia, 43600, Bangi, Malaysia 
are actually environmental risks, may have direct impact on job dispatching process of an automated MWM. In order to address this kind of risks, ROBALO framework: a Risk-Oriented joB dispAtching for mobile workforce system, has been proposed in (ShiCho et al., 2005a). ROBOLO addresses the risk of job execution for MWs and creates a balance between the cost of job's assignment and the reliability of performing a job request for MWM.

Although the environmental risks are very important, we believe that Human Recourse (HR) risks are also very crucial factors in an automated task allocation process. In our early work (Mousavi et al., 2010b) we proposed a framework for MWBS. In this framework, the life time of MWBS is divided into two phases: Initialization phase and Run-time phase. In initialization phase, which take place before starting of a period (one month in our model) of the system's run, the entire MWs of the system submit their monthly proposals to the system via a multi-agent architecture. Each monthly proposal includes number of days that an MW would be able to work during that specific month. However, MWs are subject to human factors and they might not be able to fulfill their initial proposals and claims. Therefore, the reliability of the initial plan that has to be made based on the proposals, which are acquired during the initialization phase, is not guaranteed.

Hence, in this article we have examined a specific type of HR risks called unexpected absence, which has the most important negative impact on making a monthly plan during the initialization phase of MWBS. Moreover, the effect of aforementioned risk in MWBS are shown and discussed. Furthermore, a statistical method to address this risk is formulated and the impact of the proposed method on predicting and tackling the unexpected absence is observed.

Research hypothesis: In an automated MWBS as described in (Mousavi et al., 2010b; 2011) the profit that an MW cluster earns, the penalty that it has to pay due to an unsatisfied deal and the accuracy of the brokering process is tightly coupled with the availability of the workforces as mentioned in their initial monthly proposals. Since the nature of human life is prone to unexpected and unpredictable events, there is always a gap between the claims and actual performance of an MW. Therefore, we believe that finding a way to approximately predict the aforementioned gap will lead the automated MWBS to generate a realistic initial plan, which in turn prevents the system from the risk of unexpected absence and helps the system to increase the periodical performance of the cluster in which the prediction and prevention mechanism is deployed.

Structure of the study: The remaining of this study is structured as per the following. In Materiala and Methods, mechanism and processes of gathering initial proposals from MWs used by agents involved in initialization phase of the MWBS is described, followed by explanation of the problem statement of this research. Moreover, we illustrate the proposed solution comprised of the statistical method and the incorporation of this method into a coordination mechanism, which is utilized by MWBS. The Result is devoted to data analysis, which depicts the result of applying the proposed method on a dataset collected from the past working history of a typical MW cluster. Discussion however, describes the innovative values of this research. Finally, in Conclusion we conclude this study and suggests some further works as complements to our proposed solution.

\section{MATERIALS AND METHODS}

Problem definition: Figure 1 illustrates the configuration of MWBS and agents, which are involved in Initialization phase with regards to acquisition of the monthly capacity of an MW cluster. Initialization phase starts when a Task Allocation Agent (TAA) sends a request to MWBS requesting to start creating a monthly deal. Next, Initializer Agent (IA) dispatches the initialization request to the entire Resource Agents (RA) inquiring a proposal for the coming month. Each RA then, sends a request to its corresponding MW to inquire a monthly proposal including monthly capacity, which determines the number of days that it's corresponding MW would be able to work during the next month, and the specific dates for each preferred working day. Subsequently, RA will check the proposal against a set of policy and if and only if the proposal could pass the policy checking, it would be sent back to IA as a valid proposal (Mousavi et al., 2010a; 2011).

In addition As described in (Mousavi et al., 2011), dependencies of activities in MWBS framework are managed by an Ontology Driven Coordination model (O-DC).O-DC utilizes an ontology as coordination medium called MWBSOnt represented in OWL ontology format. Therefore, the entire knowledge, which is required to perform and coordinate interdependent activities are incorporated into MWBSOnto.OWL. 
Am. J. Applied Sci., 8 (12): 1287-1294, 2011

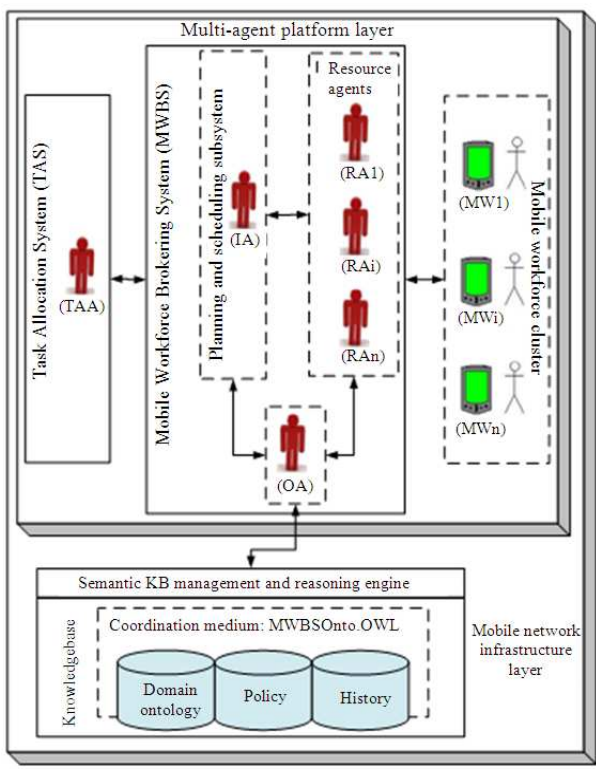

Fig. 1: MWBS in initialization phase

Although the validity of a proposal can be verified at policy checking stage, the fulfillment of the proposal still remains uncertain. Undoubtedly, the main source of uncertainty in performing a proposal in this stage is the risk of unexpected absence. Hence, a monthly deal, which is made based on uncertain data, would be insufficient.

Monthly deal: Monthly deal is the core asset of the model that we have proposed in our early works for task allocation process (Mousavi et al., 2010b; 2011). In this model, a monthly deal is important because of two main reasons. Firstly, it synchronizes the activities of different parties, which participate in this model in order to allocate the tasks to the actual resources of the MWBS (MWs). Secondly, without an appropriate monthly deal, none of these parties would be able to create a plan for the next period of the system's run. Therefore, as soon as a monthly deal is made and been agreed upon by all parties, it cannot be downgraded meaning that the capacity of the cluster, which has been mentioned in the deal cannot be decreased as any decrement in this figure will affect both the task allocation system (outsourcing vendor) as well as the client organization (service requester) (Mousavi et al., 2010b; 2011). Therefore, any decrement in a monthly deal will be penalized in order to recompense for the dissatisfaction in providing the amount of services, which have been pledged and promised by MWBS.

Nevertheless, as mentioned earlier, the risk of unexpected absence most likely does not allow a complete satisfaction in accomplishment of a monthly deal.
Problem to address: The main problem that has to be addressed in order to avoid penalties for MWBS from the one side and to evade dissatisfaction for the service requesters from the other side is: how to calculate a Realistic Monthly Capacity (RMC) for an MW cluster in presence of the risk of unexpected absence. An RMC however, has to be a symmetrical figure attempting to make a balance between profits that an MW cluster earns and penalties caused by the risk of unexpected absence. This requirement implies that if the claim of an MW needs to be modified, the modification should be based on real facts that lead the system to predict a modification ratio for the claim.

Proposed solution: As it has been described in our early works (Mousavi et al., 2010a; 2010b; 2011), there is no way to totally evade or precisely predict the risk of unexpected absence. However, an approximate prediction of the possibility of the unexpected absence for an individual MW in a specific period of time (one month in this case) is an attainable objective. For such a prediction, the closest and most trustworthy source of information undeniably is the past working history of an individual MW. Analyzing the past history of an MW, approximately illustrates that to what extend his claim is reliable. The result of such an analysis would be leading the system in generating more accurate plans for the next period of system's run.

In view of the fact that each MW cluster is compelled to a monthly deal, which is made before starting the next period, the analysis of the past history has to be focused on how precisely a monthly deal is fulfilled. Therefore, the past history of an MW should be analyzed with regard to the past monthly deals consists of the number of days that he claimed to work and the number of days that he performed for each month in the past. By analyzing these datasets, system can conclude how likely each individual MW will fulfill his obligations, which is the gap between his claims and his fulfillments. Consequently, by deducting this approximate ratio from the new proposal of an MW whose past history has been analyzed, a realistic monthly capacity can be calculated for him, which can be used to make a realistic monthly deal for his next working period. Finally, the summation of RMC's for the entire MW's of a cluster creates a suitable and more realistic norm for making a monthly deal for the next period of system's run.

In the following lines we will formulate and describe a statistical method to calculate RMC for each MW of a cluster and ORMC for the cluster itself. 
Am. J. Applied Sci., 8 (12): 1287-1294, 2011

Formulization of the solution: We formulate the statistical method to address the risk of unexpected absence of MWs in eight definitions as following:

\section{Definition 1: Let:}

Proposed $_{\mathrm{i}}$ : Be the number of working days that an MW claimed to work for month i.

Performed $\mathrm{i}_{\mathrm{i}}$ : Be the number of working days that an MW performed in month i.

D: $\quad$ Be a discreet random variable (Mendenhall et al., 2009) illustrating the difference between an MW's Proposal and Performed.

Definition 2: $\forall$ Proposed $_{i} \wedge \forall$ Performed $_{i}$ : Proposed $_{i} \geq$ Performed $_{i}$

Definition 3: $P[D=x]$ : The probability of $D$ to be equal to $\times$ (Mendenhall et al., 2009).

\section{Definition 4:}

$f\left(x_{i}\right)=P[D=x]$

$\mathrm{x}_{\mathrm{i}}=$ Proposed $_{\mathrm{i}}-$ Performed $_{\mathrm{i}}$ :

$\sum_{\mathrm{i}=1}^{\mathrm{n}} \mathrm{f}\left(\mathrm{x}_{\mathrm{i}}\right)=\sum_{\mathrm{i}=1}^{\mathrm{n}} \mathrm{p}\left[\mathrm{D}=\mathrm{x}_{\mathrm{i}}\right]$

Definition 5: Mean of the discrete random variable $\times($ Mendenhall et al., 2009):

$\mu_{\mathrm{i}}(\mathrm{n}+1)=\sum_{\mathrm{i}=1}^{\mathrm{n}} \mathrm{xif}(\mathrm{xi})=\sum_{\mathrm{i}=1}^{\mathrm{n}}(\mathrm{xi} / \mathrm{n})$

$\mu_{\mathrm{i}}(\mathrm{n}+1)=\sum_{\mathrm{i}=1}^{\mathrm{n}}[$ proposedi - performedi $\left.) / \mathrm{n}\right]$

Definition 6: Realistic Monthly Capacity of the month of $\mathrm{n}+1$ (next month) for the $\mathrm{MW}_{\mathrm{i}}$ is calculated using the following formula:

$\mathrm{PMC}_{\mathrm{i}(\mathrm{n}+1)}=$ proposed $_{\mathrm{i}}-\mathrm{u}(\mathrm{n}+1)$

Definition 7: Overall Realistic Monthly Capacity for the month of $n+1$ (next month) for entire MWs of a cluster is calculated using the following formula:

$$
\operatorname{ORMC}_{(\mathrm{n}+1)}=\sum_{\mathrm{i}=1}^{\mathrm{m}}\left(\mathrm{RMC}_{\mathrm{i}(\mathrm{n}+1)}\right)
$$

Definition 8: Ultimate Monthly Capacity (UMC) of a cluster for the month of $n+1$ (next month) is the sum of Proposed $_{i}$ of the entire MWs in the cluster. UMC for the month of $n+1$ (next month) for all MWs of a cluster is calculated using the following formula:
$\mathrm{UMC}_{(\mathrm{n}+1)}=\sum_{\mathrm{i}=1}^{\mathrm{m}}\left(\operatorname{proposed}_{\mathrm{i}(\mathrm{n}+1)}\right)$

UMC is a value that the MWBS intends to achieve but unexpected absence might not allow it to be achieved.

Incorporating the solution into O-DC: The main target of the statistical method as a contingency plan (Marshall and Alexander, 2005; 2006a; 2006b) is to synchronize the activities of the agents, which are involved in initialization phase toward making a realistic monthly deal. The contingency plan for the Risk of unexpected absence for the month of $n+1$ for $m$ agents of a cluster is defined as following:

- In Initialization phase, an Initializer Agent (IA) builds a team of the entire RAs of the system

- RAs get the proposal from their corresponding MWs

- Proposals will be checked against the policy

- If the proposal complies with the policy, then each $\mathrm{RA}_{\mathrm{i}}$ calculates the $\mathrm{RMC}_{\mathrm{i},(\mathrm{n}+1)}$

- When the entire RMCs are calculated, O-DC notifies the IA

- IA will calculate the $\mathrm{ORMC}_{(\mathrm{n}+1)}$ and $\mathrm{UMC}_{(\mathrm{n}+1)}$

- IA will send these two values to a Task Allocation Agent (TAA) and makes a deal

- The main goal of the MWBS In Run-time phse will be to fulfill the ORMC

- If ORMC is fulfilled, then the MWBS will try to fulfill UMC

- $\quad$ TAA is flexible enough to accept the increment to the initial deal, but decrement is not tolerated and will be penalized

Figure 2 shows a partial view of the MWBSOnto.OWL ontology. In this view, the relationship between a typical MW (MW1) and its pats history are depicted. MW1 is in hasHistory "objectProperty" relationship with its past histories (e.g., History_MW1_01-2010) while each past history object is in relationship with its identifying data with "DataProperties" such as hasProposal, hasRMC.

Figure 3 depicts SAPRQL query that is needed to be executed in order to retrieve the entire past history of an MW from the coordination medium. The results of this query are used to calculate the RMC for an MW for the next period. Figure 4 illustrates an INFORM KQML message that is used to add a new history (history for the next month) for an MW into the coordination medium including its proposal, date, label and its calculated RMC. 
Am. J. Applied Sci., 8 (12): 1287-1294, 2011

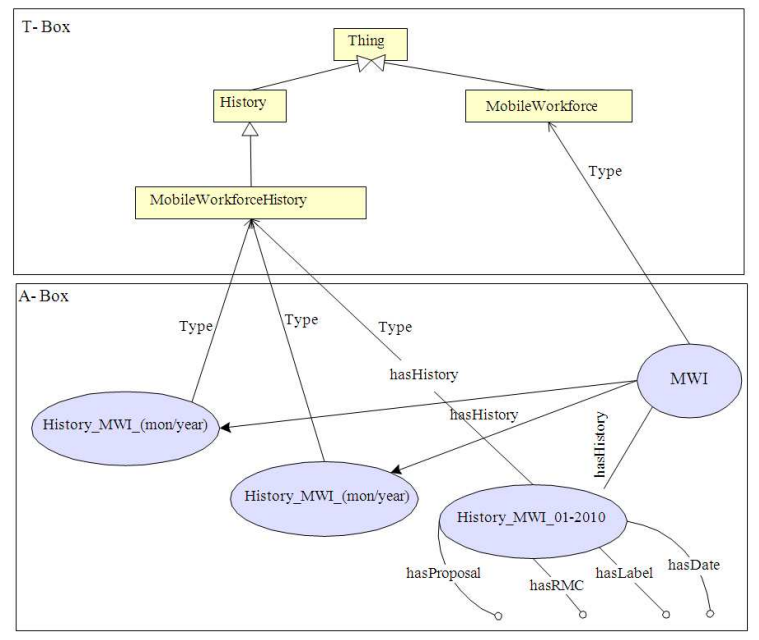

Fig. 2: Incorporating past history of MW1 into coordination medium

The SPARQL query to retrieve proposals from the

Entire Past History of MWi:

$\mathrm{Q}=$ pphRetrivalQuery:

PREFIX MWBSOnto: $<$ http://MWBSOnto.owl\#>

SELECT ?History?Proposal

WHERE \{

MWBSOnto:MWi MWBSOnto:hasHistory?History.

?History MWBSOnto:hasPorposal ?Proposal.\}

O-DS's Query execution operation:

query (pphRetrivalQuery)

Fig. 3: Query to retrieve past history for MWi from ontology

Message to add a new history for MWi:

$\mathrm{M}=$

\{ Sender: RAi, Receiver: OA, Performative:INFORM

Content:"addHistory,MWi,proposal,date,label,RMC"\}

O-DS's inform execution operation:

inform $(\mathrm{OA}, \mathrm{M})$

Fig. 4: INFORM message to add a new history for MWi into ontology

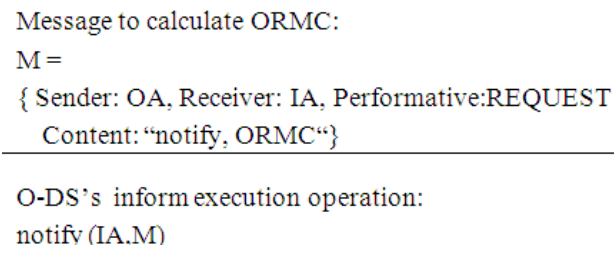

O-DS's inform execution operation:

notify (IA.M)

Fig. 5: REQUEST message to calculate ORMC
Message to add a new history for Cluster $\mathrm{Ci}$ :

$\mathrm{M}=$

\{ Sender: IA, Receiver: OA, Performative:INFORM

Content:"addHistory,Ci,proposal,date,label,ORMC"

O-DS's inform execution operation:

inform $(\mathrm{OA}, \mathrm{M})$

Fig. 6:INFORM Message to add a new History for cluster Ci into ontology

Figure 5 shows a REQUEST KQML message that is an indicator to IA agent to calculate the ORMC upon finishing the calculation of RMCs for the entire MWs. Finally Fig. 6 shows an INFORM KQML message that is used to add a new history (history of the next month) for the Cluster $\mathrm{Ci}$ ( $\mathrm{C} 1$ in this case) into the coordination medium including its proposal, date, label and its calculated ORMC.

\section{RESULTS}

Introducing datasets: The dataset being used in this study is based on the past history of a typical MW cluster called C1. As described in (Mousavi et al., 2011) and depicted in Table 1, this past history consists of 5 months of the working period (January to May 2010) for cluster C1 including 4 MWs (MW1-MW4). The main purpose of Table 1 is to illustrate how RMC for the next month of the system run (in this case June 2010) is calculated for each MW exploiting the Proposed and the Performed values of MW's past 5 months of working history using the formulas described earlier.

Calculating RMC and ORMC: Here, the execution of the formulas mentioned earlier, on the dataset shown in Table 1 is described. As stated earlier, the main purpose of these formulas is to calculate RMC for each individual MW and to calculate ORMC for the entire cluster as an end result for the process of managing the risk of unexpected absence. For this purpose, we apply the formulas on dataset for MW1 and since the mechanism is identical for every MW, we ignore applying the formula on the remaining.

The execution of formulas on MW1's past history is as following:

From definition 5 we have:

$\mu_{\mathrm{i}(\mathrm{n}+1)}=\sum_{\mathrm{i}=1}^{\mathrm{n}} \mathrm{xif}(\mathrm{xi})=\sum_{\mathrm{i}=1}^{\mathrm{n}}\left(\mathrm{x}_{\mathrm{i}=1}\right)$

$\left[\left(\operatorname{proposed}_{\mathrm{i}}-\right.\right.$ performed $\left.\left._{\mathrm{j}}\right) / \mathrm{n}\right]$ 
Am. J. Applied Sci., 8 (12): 1287-1294, 2011

Table 1: Dataset to calculate RMC and ORMC

\begin{tabular}{|c|c|c|c|c|c|c|c|c|c|c|}
\hline $\mathrm{MW}_{\mathrm{i}}$ & $\mathrm{i}$ & no & Month $_{\mathrm{no}}$ & Proposed $_{\text {no }}$ & Performed $_{n o}$ & $x_{i}$ & $\mathrm{n}$ & Proposed $\mu_{i,(n+1)}$ & $\mu_{(n+1)}$ & $\mathrm{RMC}_{\mathrm{i},(\mathrm{n}+1)}$ \\
\hline & & 1 & 10-Jan & 13 & 10 & 3 & & Proposed $_{1,6}$ & $\mu_{1,6}$ & $\mathrm{RMC}_{1,6}$ \\
\hline & & 2 & $10-\mathrm{Feb}$ & 14 & 12 & 2 & & $=$ & $=$ & $=$ \\
\hline \multirow[t]{3}{*}{$\mathrm{MW}_{1}$} & 1 & 3 & 10-Mar & 18 & 18 & 0 & 5 & 15 & 3 & 12 \\
\hline & & 4 & 10-Apr & 17 & 11 & 6 & & & & \\
\hline & & 5 & 10-May & 16 & 10 & 6 & & & & \\
\hline \multirow[t]{5}{*}{$\mathrm{MW}_{2}$} & 2 & 1 & 10-Jan & 17 & 12 & 5 & 5 & Proposed $_{2,6}$ & $\mu_{2,6}$ & $\mathrm{RMC}_{2,6}$ \\
\hline & 2 & & $10-\mathrm{Feb}$ & 14 & 14 & 0 & & $=$ & $=$ & \\
\hline & 3 & & 10-Mar & 15 & 15 & 0 & 12 & 2 & & 10 \\
\hline & 4 & & 10-Apr & 18 & 14 & 4 & & Proposed $_{3,6}$ & $\mu_{3,6}$ & $\mathrm{RMC}_{3,6}$ \\
\hline & 5 & & 10-May & 12 & 11 & 1 & & & & \\
\hline \multirow{5}{*}{$\mathrm{MW}_{3}$} & 3 & 1 & 10-Jan & 10 & 10 & & & 0 & 5 & \\
\hline & & 2 & $10-\mathrm{Feb}$ & 19 & 11 & 8 & & $=$ & $=$ & $=$ \\
\hline & & 3 & 10-Mar & 18 & 17 & 1 & & 14 & 3 & 11 \\
\hline & & 4 & 10-Apr & 16 & 10 & 6 & & & & \\
\hline & & 5 & 10-May & 12 & 11 & 1 & & & & \\
\hline \multirow[t]{6}{*}{$\mathrm{MW}_{4}$} & $4 \mu$ & 1 & 10-Jan & 15 & 10 & 5 & & Proposed $_{4,6}$ & 4,6 & $\mathrm{RMC}_{4,6}$ \\
\hline & & 2 & $10-\mathrm{Feb}$ & 18 & 16 & 2 & & $=$ & $=$ & $=$ \\
\hline & & 3 & 10-Mar & 12 & 12 & 0 & 5 & 16 & 2 & \\
\hline & & 4 & 10-Apr & 15 & 15 & 0 & & & & \\
\hline & & 5 & 10-May & 20 & 17 & 3 & & & & \\
\hline & & & & & $\mathrm{ORMC}_{6}$ & & & & & 47 \\
\hline
\end{tabular}

Table 2: Comparing proposed, RMC and performed of MWs for 4 months of data analysis period

\begin{tabular}{|c|c|c|c|c|c|c|c|c|c|c|c|c|}
\hline & \multicolumn{3}{|c|}{ June } & \multicolumn{3}{|c|}{ July } & \multicolumn{3}{|c|}{ August } & \multicolumn{3}{|c|}{ Sept } \\
\hline & Po & RMC & $\mathrm{Pe}$ & Po & RMC & $\mathrm{Pe}$ & Po & RMC & $\mathrm{Pe}$ & Po & RMC & $\mathrm{Pe}$ \\
\hline MW1 & 15 & 12 & 12 & 12 & 10 & 10 & 19 & 16 & 12 & 14 & 10 & 10 \\
\hline MW2 & 12 & 10 & 11 & 16 & 14 & 12 & 20 & 18 & 20 & 15 & 13 & 13 \\
\hline MW3 & 14 & 11 & 11 & 17 & 14 & 15 & 19 & 16 & 10 & 18 & 14 & 15 \\
\hline MW4 & 16 & 14 & 14 & 14 & 12 & 12 & 20 & 28 & 28 & 17 & 15 & 15 \\
\hline Total & 57 & 47 & 48 & 59 & 48 & 49 & 78 & 68 & 60 & 64 & 52 & 53 \\
\hline
\end{tabular}

Therefore:

$$
\begin{aligned}
& \mu 1,6=[((13-10]+(14-12)+ \\
& (18-8)+(17-11)+(16-10) / 5]=3 \\
& \mathrm{RMC}_{1.6}=\operatorname{proposed}_{1,6}-\mathrm{u} 1,6=12
\end{aligned}
$$

From definition 7 we calculate $\mathrm{ORMC}_{6}$ as follow:

$$
\begin{aligned}
& \mathrm{ORMC}_{6}=\sum_{\mathrm{i}=1}^{\mathrm{n}}\left(\mathrm{RMC}_{\mathrm{i},(\mathrm{n}+1)}\right) \\
& \operatorname{and}(\mathrm{m}=4)=12+10+11+14=47
\end{aligned}
$$

\section{DISCUSSION}

the effectiveness and accuracy of our proposed model in predicting actual capacity of the workforces in presence of unexpected absence is investigated and portrayed. Our discussion is based on comparison between 3 main variables that determines the effect of unavailability of the MWs namely: Po (proposed), Pe (performed) and the RMC.

Table 2 includes the values of the abovementioned variables for $4 \mathrm{MWs}$ of a typical cluster $(\mathrm{C} 1)$ for the period of 4 months (June to September 2010). In this table RMCs are calculated exploiting the methods described earlier and the data shown in Table 1 presenting the past history of the cluster for the past 5 consecutive months. The last row of Table 2 contains the total values of Po, RMC and Pe for the entire MWs in each month, not to mention that the total RMC is actually the ORMC of the cluster.

Table 3 On the other hand, depicts the overall value of Po, RMC and Pe for each individual MW based on the data taken from the rows of the Table 2. In addition, difference between overall proposed and overall performed (DFP) and difference between overall proposed and overall RMC (DFRMC) for each individual MW for 4 months of data analysis period are calculated and shown. Next, DFP and DFRMC are used to figure out the improvement in predicting the actual performed for each individual MW, which are shown in a column titled as Individual Improvement. Finally the last row of Table 3 illustrates the average overall improvement in predicting the actual performed value for the cluster $\mathrm{C} 1$ in four consecutive months, which is a strongly considerable amount of $86 \%$. 
Am. J. Applied Sci., 8 (12): 1287-1294, 2011

Table 3: Illustration of improvement in predicting the risk of unexpected absence

\begin{tabular}{llllllr}
\hline & Overall proposed & Overall performed & Overall RMC & DFP & DFRMC & Improvement individual (\%) \\
\hline MW1 & 60 & 44 & 48 & 16 & 4 & 75 \\
MW2 & 63 & 56 & 55 & 7 & 1 & 86 \\
MW3 & 68 & 51 & 54 & 17 & 3 & 82 \\
MW4 & 67 & 59 & & 8 & 0 & 100 \\
Average Overall Improvement: & & & & & 86 \\
\hline
\end{tabular}

Table 4: Comparing total values of proposed ORMC and performed for each individual month of data analysis period

\begin{tabular}{lllll}
\hline & June & July & August & September \\
\hline Total proposed & 57 & 59 & 78 & 64 \\
ORMC & 47 & 48 & 68 & 52 \\
Total performed & 48 & 49 & 60 & 53 \\
\hline
\end{tabular}

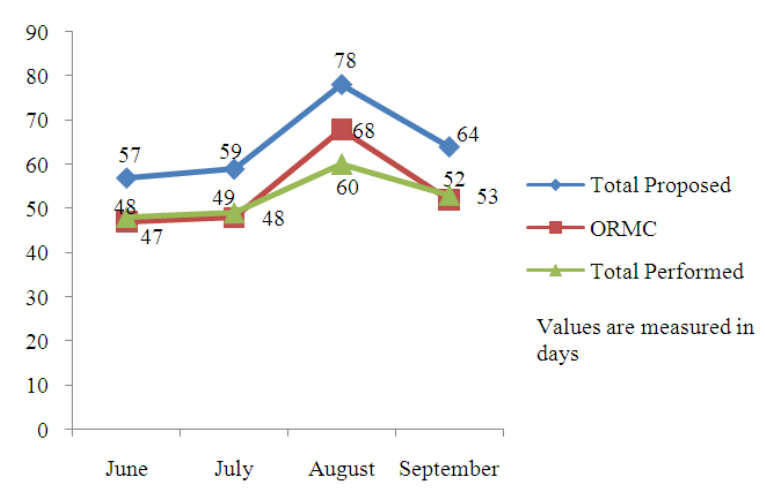

Fig. 7: Overall comparison between proposed, ORMC and performed for 4 months

Furthermore, In Table 4, Total Performed, ORMC and Total Proposed for cluster $\mathrm{C} 1$ for each individual month of the data analysis period are shown. These values are portrayed in Fig. 7 as line charts each one which represents one of these total values.

It can be clearly seen in Fig. 7 that the ORMC and performed lines are considerably closed to each other and they even converge in most of the cases, while total proposed line is always far from total performed. The convergence and the closeness of ORMC and total performed line depicts the fact that our proposed statistical method is an efficient and satisfactory method to predict and calculate a realistic figure for the monthly working capacity of the MWs of a cluster, which is significantly close to the actual performance of the MWs.

\section{CONCLUSION}

In conclusion, the result of this research proves that ontology and semantic knowledge representation and reasoning techniques can be used as effective tools to empower agents who are situated in dynamic environments in performing their tasks, as well as in communicating with each other, in a robust and acceptable manner. Multi-agent systems which are implemented using this approach are more generic, adaptable and consistent in comparison with other approaches.

In this study we have proposed and formulated a statistical method to address a specific type of human resource risk management called unexpected absence for mobile workforces. The proposed model is based on evaluation of the past working history of t the mobile workforces of an MWBS cluster. The main objective of the proposed model from the one side is to prevent the penalty by predicting the possible absence of MWs and from the other side to make a balance between penalty and profits that a cluster may pay or earn by increasing the accuracy of the prediction. Moreover, since MWBS utilizes a multi-agent architecture and knowledge being used by MWBS's agents is stored in an ontology, which represents a coordination medium, the past history of the system is incorporated into the ontology as well. Furthermore, the mechanism to retrieve required knowledge and the coordination operations and primitives to manipulate the knowledge are described in this study. Finally, the proposed method has been applied on a dataset including the past history of the system run for the 5 consecutive months and the behavior of the system has been observed and analyzed for 4 months. The results of our observation and analysis reveal that the proposed method increases the overall accuracy of predicting the unexpected absence for a typical cluster during the 4 months of data analysis period by $85 \%$ in average. This rate is a considerable figure that proves the efficiency and applicability of the proposed method.

However, despite of the proven efficiency of the proposed method, there are many other factors that can be added to the formal method in order to increase the accuracy in predicting the aforementioned risk. Some of these factors are: marital statues, number of children, the health record and the family health record of MWs. By analyzing these types of information, which are generally the cause of the unexpected absence, system can detach and prevent the risk in a more accurate and reliable manner. Therefore, it is strongly suggested for the future research works in this field. 


\section{REFERENCES}

Chiu, D.KW., S.C. Cheung and L. Ho-fung, 2005. A Multi-agent infrastructure for mobile workforce management in a service oriented enterprise. Proceedings of the 38th Hawaii International Conference on System Sciences, Jan. 3-6, IEEE Xplore Press, Hawaii, pp: 85c-85c. DOI: 10.1109/HICSS.2005.28

Kappel, G., S. Rausch and W. Retschitzengger, 1998. Coordination in workflow management systems-A rule-based approach. Coordinat. Technol. Collaborative Appli. Lecture Notes Comput. Sci., 1364: 99-119. DOI: 10.1007/BFb0027102

Malone, T.W and K. Crowston, 1990. What is coordination theory and how can it help design cooperative work systems. Proceedings of the Conference on Computer Support Cooperative Work, Oct. 7-10, ACM Press, Los Angeles, CA., USA., pp: 357-370. DOI: 10.1145/99332.99367

Marshall, M.I. and C. Alexander, 2006a. Using a contingency plan to combat human resource risk. Online Journal of Purdue Extension.

Marshall, M.I. and C. Alexander, 2005. Planning for the unexpected human resource risk and contingency planning. Journal of Purdue Extension EC-736.

Marshall, M.I. and C. Alexander, 2006b The Risk Matrix: Illustrating the Importance of Risk Management Strategies. Online Journal of Purdue Extension.

Meijer, G.R., J. Samuels and F. Terpstra, 2002. Modeling user acceptance and technology adoption: Is there a case for value added services. http://staff.science.uva.nl/ ftrpstra/its-2002.pdf

Mendenhall, W., R.J. Beaver and B.M. Beaver, 2009. Introduction to Probability and Statistics. 13th Edn., Brooks/Cole Cengage Learning, Canada, ISBN: 9780495389538 , pp: 746.

Mousavi, A. and M.J. Nordin 2007. An architectural model for a multi-agent mobile workforce brokerage system based on CBR-BDI agent architecture and active shared-data space coordination model. Proceedings of the IEEE International Conference on Electrical Engineering and Informatics, Jun. 17-19, Bandung Indonesia, pp: 294-297.
Mousavi, A., M.J. Nordin and Z.A. Othman, 2010a. An ontology driven, multi-agent based framework for automated resource allocation in mobile workforce management systems. Proceedings of 4th IEEE International Symposium on Information Technology, Jun. 15-17, IEEE Xplore Press, Kuala Lumpur, Malaysia, pp: 1416-1421. DOI: 10.1109/ITSIM.2010.5561520

Mousavi, A., M.D.J. Nordin and Z.A. Othman, 2010b. An ontology driven, procedural reasoning systemlike agent model, for multi-agent based mobile workforce brokering systems. J. Comput Sci., 6: 557-565. DOI: $10.3844 /$ jcssp.2010.557.565

Mousavi, A., M.J. Nordin and Z.A. Othman, 2011. Ontology-driven coordination model for multiagent-based mobile workforce brokering systems. J. Applied Intell. DOI: 10.1007/s10489011-0294-z

Pal, S.K., T.S. Dillon and D.S. Yeung, 2001. Soft Computing in Case based Reasoning. 1st Edn., Springer, London, New York, ISBN: 185233262X, pp: 372.

Shi-Cho, C., T. Hung-Wen, H. Chih-Hao, L. HanChao and T. Tse-Ming et al., 2005a. Take risks into consideration while job dispatching. IFIP Int. Federation Inform. Process., 191: 1-14. DOI: 10.1007/0-387-31166-1_1

Shi-Cho, C., T. Hung-Wen, L. Han-Chao, T. Tse-Ming and L. Raymund, et al., 2005b. Robalo: A riskoriented job dispatching mechanism for workforce management system. IFIP Int. Federation Inform. Process., 189: 559-570. DOI: 10.1007/0-38729773-1_37

Wang, Y., E.V.D. Kar, G. Meijer and M. Hunteler, 2005. Improving business processes with mobile workforce solutions. Proceedings of the International Conference on Mobile Business, July 11-13, IEEE Xplore Press, pp: 94-102. DOI: 10.1109/ICMB.2005.46

Wang, Y., D.K. Van, E. Meijer and G.R.H.G. Sol, 2006. Business engineering with mobile workforce solutions. Int. J. Comput. Syst. Signals, 7: 42-54. 